\title{
Corticospinal excitability of tibialis anterior and soleus differs during passive ankle movement
}

\author{
Jakob Škarabot ${ }^{1}$ (D) Paul Ansdell ${ }^{1} \cdot$ Callum G. Brownstein $^{1,2} \cdot$ Kirsty M. Hicks $^{1} \cdot$ Glyn Howatson $^{1,3} \cdot$ Stuart Goodall $^{1}$. \\ Rade Durbaba ${ }^{1}$
}

Received: 8 April 2019 / Accepted: 20 June 2019 / Published online: 26 June 2019

(c) The Author(s) 2019

\begin{abstract}
The purpose of this study was to assess corticospinal excitability of soleus (SOL) and tibialis anterior (TA) at a segmental level during passive ankle movement. Four experimental components were performed to assess the effects of passive ankle movement and muscle length on corticospinal excitability (MEP/ $\left.M_{\max }\right)$ at different muscle lengths, subcortical excitability at the level of lumbar spinal segments $\left(\mathrm{LEP} / M_{\max }\right.$ ), intracortical inhibition (SICI) and facilitation (ICF), and $H$-reflex in SOL and TA. In addition, the degree of fascicle length changes between SOL and TA was assessed in a subpopulation during passive ankle movement. Fascicles shortened and lengthened with joint movement during passive shortening and lengthening of SOL and TA to a similar degree $(p<0.001)$. Resting motor threshold was greater in SOL compared to TA $(p \leq 0.014)$. $\mathrm{MEP} / M_{\max }$ was facilitated in TA during passive shortening relative to the static position $(p \leq 0.023)$ and passive lengthening $(p \leq 0.001)$, but remained similar during passive ankle movement in SOL $(p \geq 0.497)$, regardless of muscle length at the point of stimulus $(p=0.922)$. LEP/M $M_{\max }$ (SOL: $p=0.075$, TA: $\left.p=0.071\right)$, SICI (SOL: $p=0.427$, TA: $p=0.540$ ), and ICF (SOL: $p=0.177$, TA: $p=0.777)$ remained similar during passive ankle movement. $H$-reflex was not different across conditions in TA $(p=0.258)$, but was reduced during passive lengthening compared to shortening in SOL $(p=0.048)$. These results suggest a differential modulation of corticospinal excitability between plantar and dorsiflexors during passive movement. The corticospinal behaviour observed might be mediated by an increase in corticospinal drive as a result of reduced afferent input during muscle shortening and appears to be flexor-biased.
\end{abstract}

Keywords Ia afferent $\cdot$ Fascicle length $\cdot H$-reflex $\cdot$ Transcranial magnetic stimulation

\section{Introduction}

Corticospinal excitability is constantly modulated during passive and active movements. Isotonic movements modify corticospinal excitability, such that excitability tends to be lower during lengthening relative to shortening and isometric contractions (Abbruzzese et al. 1994; Gruber et al. 2009;

Rade Durbaba

rade.durbaba@northumbria.ac.uk

$1 \quad$ Faculty of Health and Life Sciences, Northumbria University, Newcastle upon Tyne, England NE1 8ST, UK

2 Univ Lyon, UJM-Saint-Etienne, Laboratoire Interuniversitaire de Biologie de la Motricité, EA 7424, 42023 Saint-Étienne, France

3 Water Research Group, School of Environmental Sciences and Development, Northwest University, Potchefstroom, South Africa
Duclay et al. 2014), which seems to depend on the amount of Ia afferent feedback (Doguet et al. 2017). However, elucidating the direct effect of muscle length-related feedback on the corticospinal tract output during dynamic contractions is challenging due to the influence of postsynaptic control mechanisms (Valadão et al. 2018; Barrué-Belou et al. 2018), and potential differences in neural drive that can influence neurophysiological responses (Abbruzzese et al. 1994; Morita et al. 2000).

Potential insight into the effect of muscle length-related feedback on the corticospinal response might be gained by assessing responses during passive movement. With passive muscle lengthening, the firing of muscle spindle afferents increases proportionally to the magnitude of the stretch, but remains low during shortening of a muscle (Matthews 2011; Day et al. 2017). This behaviour at the somatosensory receptor level might, in turn, modulate the corticospinal responses. Indeed, corticospinal excitability has been shown 
to be reduced during passive lengthening of the wrist flexors and extensors, and has been related to the degree of muscle spindle afferent feedback (Lewis et al. 2001; Lewis and Byblow 2002; Coxon et al. 2005). Notwithstanding these findings, the level of neural axis at which afferent-mediated changes in corticospinal output occur has not been elucidated. From a cortical perspective, intracortical inhibition is modulated during passive shortening and lengthening of the upper limbs (Lewis et al. 2001). However, despite the presence of a facilitatory corticospinal response during passive shortening of upper limb muscles (Chye et al. 2010), the contribution of intracortical facilitatory circuits to augmented corticospinal excitability has not been considered. In addition, passive lengthening of soleus (SOL) has been shown to be accompanied by greater presynaptic inhibition (Pinniger et al. 2001), whilst less is known about the effect of passive movement on subcortical output of the corticospinal tracts, which are likely devoid of classical presynaptic influence (Nielsen and Petersen 1994). In addition, far less is known about corticospinal excitability during passive movement of the lower limbs, which might differ due to disparities between facilitatory and inhibitory intracortical outputs and corticospinal projections to upper and lower limb muscles (Brouwer and Ashby 1990; Chen et al. 1998).

The SOL and tibialis anterior (TA) muscles are integral for movement about the ankle joint. For example, SOL plays a crucial role in balance (Capaday et al. 1999), whereas TA is involved in the control of foot drop during heel strike and foot lift during the swing phase (Byrne et al. 2007), as well as toe clearance through the gait cycle (Nielsen et al. 2003). The SOL and TA muscles also exhibit distinct roles in quiet standing and postural sway, with the former acting as agonist and the latter providing the proprioceptive feedback via reciprocal inhibition (Di Giulio et al. 2009). Due to these differences in function, TA and SOL might require distinct corticospinal control. From a neural perspective, TA and SOL have been shown to exhibit differences in the quantity of muscle spindles that affects the relative input from Ia afferents (Banks 2006; De Luca and Kline 2012), the type and the size of motor units (Burke 1967; Dum and Kennedy 1980), reciprocal spindle afferent input (Yavuz et al. 2018), distribution of direct corticomotoneuronal projections (Brouwer and Ashby 1992; Brouwer and Qiao 1995), intracortical inhibition (Lauber et al. 2018), and preferences in the input from pyramidal tract into the spinal network (Brooks and Stoney 1971).

The aim of this study was to investigate corticospinal function of TA and SOL during passive ankle movement. Four experimental components were performed designed to assess (1) corticospinal modulation at different muscle lengths; (2) the contribution of cortical neurons and spinal motoneurons to the corticospinal response: (3) intracortical facilitation and inhibition; and (4) the contribution of Ia afferent input to spinal motoneurons in quiescent SOL and TA during passive ankle movement. It was hypothesised that corticospinal excitability will be dependent on the change in muscle length and muscle studied, and will be attributable to processes at both cortical and spinal levels.

\section{Methods}

\section{Participants}

Twenty healthy, volunteers $(25 \pm 4$ years, $175 \pm 9 \mathrm{~cm}$, $78.9 \pm 16.8 \mathrm{~kg}$; 9 females) participated in the study. Based on the previous studies (Lewis et al. 2001; Lewis and Byblow 2002), an a priori power analysis (Faul et al. 2007) showed that six participants were needed to observe modulation of MEP amplitude with passive movement. To reduce the potential influence of female sex hormones on TMSevoked responses, all females were tested in the early follicular phase of the menstrual cycle where both oestrogen and progesterone concentrations are likely to be low (Elliott et al. 2003) or whilst taking oral contraceptives (Ansdell et al. 2019). All participants were free from neurological illness or musculoskeletal injury, were not taking any medications known to affect the nervous system, and reported no contraindications in TMS safety screening (Keel et al. 2001). The study conformed to the standards of Declaration of Helsinki, apart from pre-registration in a database. All procedures were approved by Northumbria University Ethics Committee (BMS57UNNJSRD2016). All participants provided written informed consent prior to the start of the study proceeding.

\section{Experimental design}

The study involved four experimental components designed to investigate the effect of passive ankle motion on corticospinal excitability at different muscle lengths (Experiment 1), corticospinal and spinal motoneuron excitability (Experiment 2), intracortical facilitation and inhibition (Experiment 3), and the contribution of Ia afferent input to spinal motoneurons (Experiment 4) in resting SOL and TA. Twelve participants took part in Experiment 1 ( $26 \pm 4$ years, $176 \pm 9 \mathrm{~cm}, 77.8 \pm 16.8 \mathrm{~kg} ; 6$ females). In Experiment 2, two participants did not return for further testing due to scheduling conflicts, and an additional participant was recruited ( $n=11 ; 26 \pm 4$ years, $178 \pm 8 \mathrm{~cm}, 81.6 \pm 16.2 \mathrm{~kg} ; 5$ females). Due to larger heterogeneity of responses, additional participants were recruited for Experiment $3(n=15 ; 25 \pm 4$ years, $178 \pm 9 \mathrm{~cm}, 83.1 \pm 17.1 \mathrm{~kg} ; 5$ females). In Experiment 4, obtaining $H$-reflexes in resting TA proved challenging as has been previously reported (Roy and Gorassini 2008; Burke 2016). After screening 24 individuals, only five participants 
exhibited clear and consistent $H$-reflexes in quiescent TA to allow for comparison with SOL and took part in Experiment 4 ( $24 \pm 3$ years, $176 \pm 11 \mathrm{~cm}, 72.2 \pm 14.3 \mathrm{~kg}$; 1 female $)$. Individuals that took part in all four experiments were tested within 6 weeks of the first visit to the laboratory.

\section{Procedures}

\section{Experimental setup}

Participants sat on an isokinetic dynamometer (Cybex, Lumex Inc., USA) with hip and knee at $60^{\circ}$ and $90^{\circ}$ flexion, respectively. All testing was performed on the dominant limb as determined by the lateral preference inventory (Coren 1993). The foot was strapped securely to a metal foot plate attached to the lever arm of the motor with a velcro strap. The range of motion of the device was set to $20^{\circ}$, ranging from $10^{\circ}$ plantar flexion to $10^{\circ}$ dorsiflexion with anatomical zero being when the ankle was set at $90^{\circ}$. During passive ankle motion, the motor of the device moved the foot plate throughout the range of motion at $5^{\circ} \mathrm{s}^{-1}$. TMS or electrical stimulation was delivered at anatomical zero (considered intermediate muscle length) during static position and passive ankle movement. In addition, stimuli were delivered at $\pm 7.5^{\circ}$ relative to anatomical zero in the part of the study examining corticospinal responses at different muscle lengths during passive ankle movement, with positive and negative degree values indicating plantar and dorsiflexion, respectively. Thus, at positive values relative to anatomical zero, the muscle was at longer and shorter length for TA and SOL, respectively, and vice versa for negative values. Based on the joint angles and movement velocity, the stimuli were delivered $2 \mathrm{~s}$ (Experiment 1-4), and 0.5 and $3.5 \mathrm{~s}$ after the onset of movement (Experiment 1). To minimise thixotropic effect on the responses, participants were resting in the starting position at least $10 \mathrm{~s}$ before the start of passive motion (Proske et al. 1993). At least $15 \mathrm{~s}$ of rest was employed before each motion.

\section{Electromyography}

Electromyographic (EMG) activity was recorded with a bipolar electrode arrangement $(8 \mathrm{~mm}$ diameter, $20 \mathrm{~mm}$ inter-electrode distance; Kendall 1041PTS, Tyco Healthcare Group, USA) over the muscle belly of SOL and TA with the reference electrode placed over the medial malleolus according to SENIAM recommendations (Hermens et al. 2000). For SOL, the electrodes were positioned at two-thirds of the line between the medial condyle of the femur to the medial malleolus. For TA, the electrodes were placed at one-third of the length between the tip of the fibula and the tip of the medial malleolus. Prior to placement of electrodes, the recording site was shaved, abraded with preparation gel, and wiped clean with an alcohol swab to ensure appropriate impedance $(<2 \mathrm{k} \Omega)$. The EMG signal was amplified $(1000 \times)$, band pass filtered $(20-2000 \mathrm{~Hz}$; Neurolog System, Digitimer Ltd, UK), digitised (5 kHz; CED 1401, CED, UK), acquired, and analysed off line (Spike2, v8, CED, UK).

\section{Transcranial magnetic stimulation}

Single- and paired-pulse TMS were delivered using two Magstim $200^{2}$ magnetic stimulators (Magstim Co., Ltd., Whitland, UK) via a concave double-cone coil. The coil was positioned over the leg area of the primary motor cortex contralateral to the target dominant leg and was oriented to induce posterior-to-anterior cortical current. Whilst corticospinal responses might differ between the dominant and non-dominant hemisphere in the upper limbs, evidence is lacking that a similar difference exists for lower limbs (Smith et al. 2017). Initially, the centre of the coil was placed $1 \mathrm{~cm}$ lateral and posterior to the vertex (Devanne et al. 1997), after which it was moved medio-laterally and posterior-anteriorly in small steps around the initial position until the spot consistently evoking the greatest MEP in the target muscle, i.e., SOL or TA, was identified (hotspot). Once identified, the back of the coil was marked directly on the scalp to ensure consistent placement throughout the trial. Resting motor threshold (rMT) was then established with the ankle positioned at anatomical zero and determined as the intensity that elicited an MEP amplitude $\geq 50 \mu \mathrm{V}$ in 3 out of 5 trials (Rossini et al. 1994). The hotspot and rMT were determined separately for SOL and TA, and separately during each experimental session.

\section{Lumbar-evoked potentials}

Lumbar-evoked potentials (LEPs) were elicited with a constant-current stimulator (1 ms pulse duration; Digitimer DS7AH, Hertfordshire, UK) to assess spinal motoneuronal excitability during passive movement of the ankle. The cathode was centred over the first lumbar spinous process $(5 \times 9 \mathrm{~cm}$; Nidd Valley Medical Ltd., Bordon, UK) with the long axis of the electrode aligned to the centre of the vertebral column. The surface area of the cathode covered two spinous processes above and below the centre point $\left(T_{11}-L_{3}\right)$. A cathode of large area was chosen as it produced less discomfort and greater tolerance by participants (Ugawa et al. 1995; Kuhn et al. 2010). The anode $\left(2.5 \mathrm{~cm}^{2}\right)$ was placed $5 \mathrm{~cm}$ above the upper edge of the cathode (Ugawa et al. 1995), corresponding to the level of the eighth thoracic spinous process $\left(T_{8}\right)$. This stimulating site has recently been shown to activate corticospinal axons at the level of lumbar spinal segments ( ̌̌karabot et al. 2018). 


\section{Percutaneous nerve stimulation}

Percutaneous nerve stimulation (1 ms pulse duration; Digitimer DS7AH, Hertfordshire, UK) was performed to elicit $H$-reflexes in Experiment 4 in SOL and TA (see 'Experiment 4 ' for a more detailed procedure). To account for changes at the skin-electrode surface, maximal compound action potentials $\left(M_{\max }\right)$ were elicited in SOL and TA and subsequently used for normalisation of the responses across Experiments $1-4$. To evoke responses in SOL, the cathode $\left(2.5 \mathrm{~cm}^{2}\right.$; Nidd Valley Medical Ltd., Bordon, UK) was placed over the tibial nerve in the popliteal fossa with the anode $(5 \times 9 \mathrm{~cm})$ positioned over the patella. To elicit responses in TA, a $40 \mathrm{~mm}$ cathode/anode arrangement (Digitimer, Hertfordshire, UK) was placed over the common peroneal nerve below the head of the fibula. $M_{\max }$ was elicited separately for SOL and TA by gradually increasing the intensity of percutaneous stimulation until the EMG response plateaued, upon which the intensity was further increased by $30 \%$. In Experiments 1-4, four stimuli eliciting $M_{\max }$ in both muscles were delivered at anatomical zero. In addition, in Experiment 1, four $M_{\max }$ were elicited at $\pm 7.5^{\circ}$ relative to anatomical zero. Since $M_{\max }$ is sensitive to changes in static positions (Gerilovsky et al. 1989), but not shortening and lengthening when stimuli are delivered at the same joint angle (Pinniger et al. 2001), $M_{\max }$ was elicited only during static positions.

\section{Experimental procedures}

The experimental procedures are summarised in Fig. 1.

\section{Assessment of fascicle length changes during passive ankle movement}

Changes in joint angle during passive movement of muscle are usually assumed to reflect changes in the total muscle-tendon unit length. However, the proprioceptive feedback originating from muscle spindles is more closely

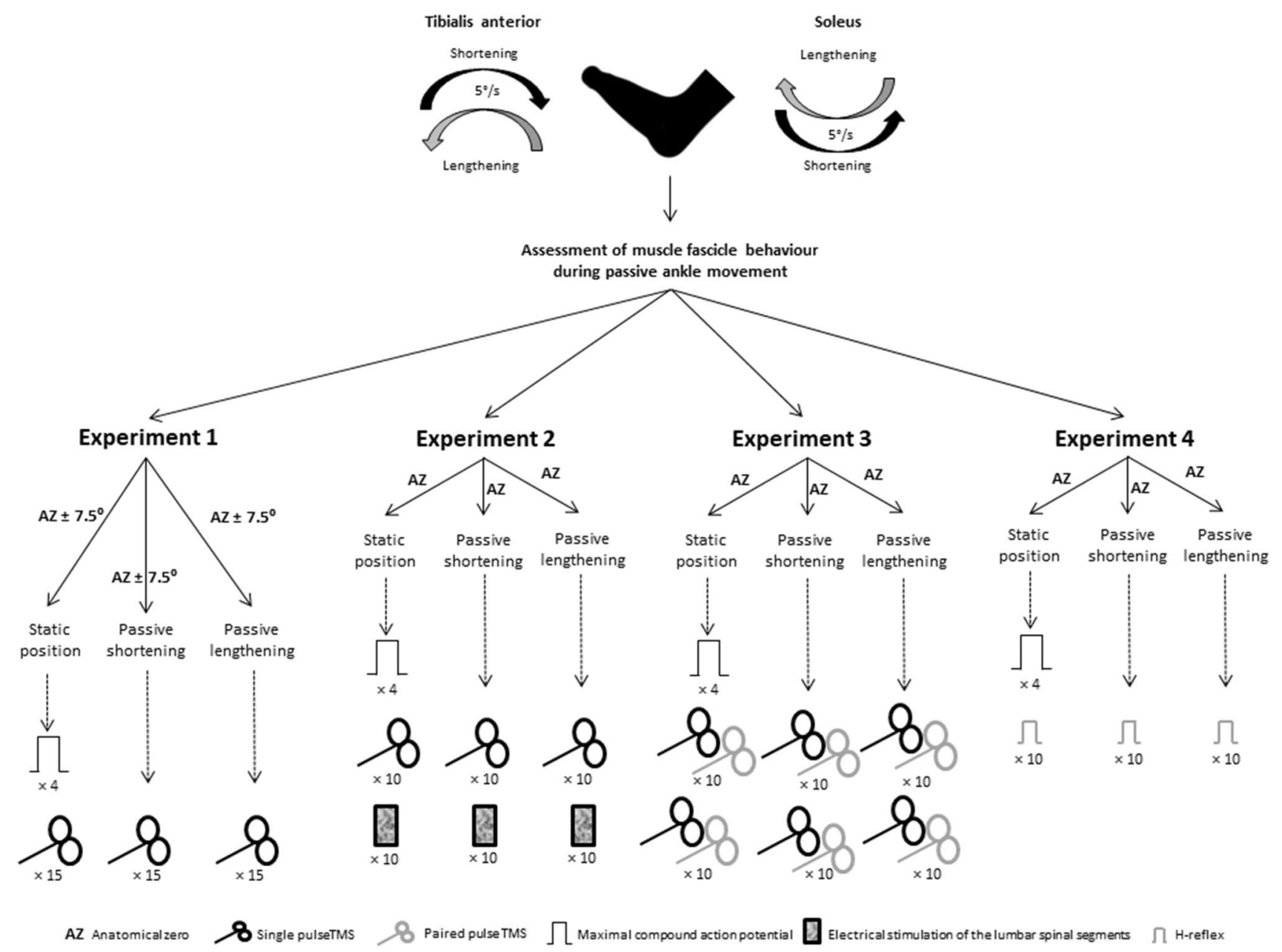

Fig. 1 An overview of experimental procedures 
related to changes in fascicle length than joint angle (Matthews and Stein 1969; Morgan et al. 2000; Day et al. 2017). As modulation of corticospinal excitability has been linked to afferent feedback pertaining to changes in muscle length (Lewis et al. 2001; Lewis and Byblow 2002; Coxon et al. 2005), it is important to establish whether changes in joint angle correspond to changes in fascicle length. Furthermore, it is important to assess the similarity of those changes between TA and SOL to ensure that the corticospinal responses are not confounded by differing magnitude of afferent feedback between the two muscles.

In a subpopulation of seven individuals $(27 \pm 3$ years, $179 \pm 8 \mathrm{~cm}, 84.1 \pm 19.6 \mathrm{~kg} ; 3$ females), fascicle behaviour of the SOL and TA during $20^{\circ}$ of passive ankle movement at $5^{\circ} \mathrm{s}^{-1}$ was tracked using ultrasound. Ultrasound (AU5 Harmonic, Esatoe Biomedica, Genoa, Italy) images were captured in real time (25 Hz sampling; AVer Media Capture Studio, AVer Media Technologies, New Taipei City, Taiwan). After identification and marking of the proximal and distal insertion of the muscle, a B-mode linear array probe $(7.5 \mathrm{MHz}, 55 \mathrm{~mm}$ width) was held with constant light pressure, perpendicular to the dermal surface along the midsagittal plane of the muscle. For SOL, the probe was positioned at $50 \%$ of the distance between the popliteal crease and the lateral malleolus (Valadão et al. 2018). In three participants, this position had to be adjusted to $30 \%$ of the same reference line to allow for clear imaging of the fascicles (Valadão et al. 2018). For TA, the probe was positioned between the fibular head and medial malleolus (Bland et al. 2011) at the site corresponding to the thickest portion of the muscle as identified by the ultrasound (Reeves and Narici 2003). A hypoallergenic ultrasound gel (Parker, Park Laboratories Inc., Fairfield) was used to enhance coupling between the skin and the probe. An echo-absorptive marker was placed between the skin and the probe to ensure the probe did not move during the recording. An externally generated square wave pulse was used to synchronise the ultrasound images with the dynamometer position acquisition system. Framecapture software (Adobe Premier Elements, version 15) was used to acquire ultrasound images, corresponding to every $0.5^{\circ}$ of ankle angle, for offline analysis. Using digitising software (Image J 1.45, National Institutes of Health, USA), SOL and TA fascicle length was measured at full ROM ( $\pm 10^{\circ}$ relative to anatomical zero) and the positions corresponding to where stimulations were delivered (anatomical zero and $\pm 7.5^{\circ}$ relative to anatomical zero). Fascicle length was measured from the visible insertion of the fibre between the deep and superficial aponeurosis for SOL (Valadão et al. 2018), and from central to the superficial aponeurosis for TA (Fig. 2; Reeves and Narici 2003). The fascicle was measured if it remained visible across the entire ultrasound image. Where the fascicle extended beyond the ultrasound image, linear continuation of the fascicle and aponeurosis was assumed ( $\mathrm{ICC}=0.853$, Ando et al. 2014; $2.4 \%$ error rate, Reeves and Narici 2003). To reduce error associated with estimation of fascicle length, an average of three fascicles across the image was taken (Guilhem et al. 2011).

\section{Experiment 1: corticospinal responses at different muscle lengths during passive ankle movement}

Responses in 12 individuals were assessed across nine conditions: static position and passive shortening and lengthening with single-pulse TMS delivered at anatomical zero (intermediate muscle length) and at $\pm 7.5^{\circ}$ relative to anatomical zero (shorter and longer muscle length depending on the muscle as explained above). The order of conditions was randomised. Intensity of TMS was standardised to $1.2 \times \mathrm{rMT}$
Fig. 2 An example of ultrasound sagittal plane scans. Images were taken at anatomical zero and show the fascicle length (Lf) measured from the visible insertion of the fibre between the deep and superficial aponeurosis in soleus (left panel), and from central to the superficial aponeurosis tibialis anterior (right panel). The shadow in the images represents the echo-absorptive marker used to ensure no movement between the skin and the probe occurred throughout ankle movement

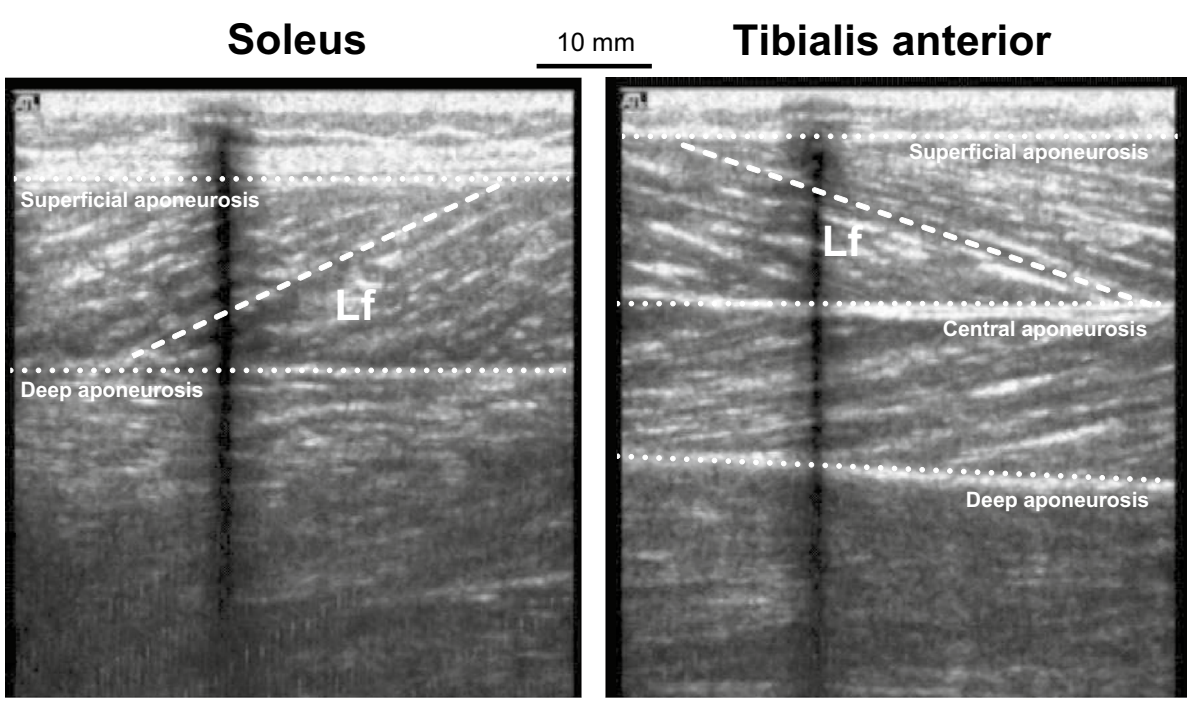


in the static position, as this intensity corresponds with the ascending limb of the stimulus-response curve (Han et al. 2001), making the responses susceptible to changes with passive ankle movement. A total of 15 MEPs were elicited in each condition.

\section{Experiment 2: corticospinal and spinal motoneuronal responses during passive ankle movement}

In eleven individuals, ten LEPs and ten MEPs were evoked during static position and passive ankle movement in SOL and TA (randomised order). The intensity of TMS was standardised to $1.2 \times$ rMT. Pilot testing indicated that MEPs elicited at $1.2 \times \mathrm{rMT}$ in the resting position evoke a response of $\sim 5-10 \% M_{\max }$. Thus, the stimulus intensity of LEPs was standardised to elicit a response of $\sim 5-10 \% M_{\max }$ in the resting position (current intensity: $151 \pm 54$ and $163 \pm 54 \mathrm{~mA}$ for SOL and TA, respectively). All stimuli were delivered at anatomical zero.

\section{Experiment 3: intracortical inhibition and facilitation during passive ankle movement}

In 15 participants, paired-pulse paradigms (SICI and ICF) were employed during static position and passive movement of the ankle to elicit responses in SOL and TA (randomised order). The TMS configuration used consisted of conditioning stimuli of 0.7 and $0.6 \times \mathrm{rMT}$ and ISIs of 2 and $10 \mathrm{~ms}$ for SICI and ICF, respectively (Brownstein et al. 2018). The test stimulus was always delivered at $1.2 \times \mathrm{rMT}$. Ten unconditioned and ten conditioned pulses were delivered in an alternating fashion for each paired-pulse paradigm at anatomical zero.

\section{Experiment 4: $\mathrm{H}$-reflex during passive ankle movement}

In five participants, $H / M$ recruitment curves were first constructed in the anatomical zero position in both SOL and TA by gradually increasing the intensity of stimulation by $0.3 \mathrm{~mA}$ every three pulses from $H$-reflex threshold to $M_{\max }$. Recruitment curves were obtained only in the static position since only the amplitude of the $H$-reflex, but not the slope of the $H / M$ curve differs between passive shortening and lengthening (Pinniger et al. 2001). The $H$-reflex amplitude was evoked with a small $M$-wave of consistent size across conditions (SOL: $12 \pm 6 \% M_{\max }$, TA: $8 \pm 2 \% M_{\max }$; $p=0.21$ ), ensuring that the same proportion of motor units were activated across conditions (Duclay and Martin 2005), and that the $H$-reflex was produced on the ascending limb of the $H / M$ recruitment curve and was, thus, susceptible to a change with passive ankle movement (Pierrot-Deseilligny and Burke 2005). Ten $H$-reflexes were elicited in SOL and TA during static position and passive ankle movement in a randomised order. All stimuli were delivered at anatomical zero. Recordings were made separately for TA and SOL.

\section{Data analyses}

EMG activity was visually inspected during the experiments to ensure that participants maintained a relaxed muscle. If voluntary EMG activity was observed, the trial was discarded and additional trials were performed. Furthermore, root-mean-square EMG activity $\left(\mathrm{RMS}_{\mathrm{EMG}}\right.$ ) was measured $100 \mathrm{~ms}$ prior to each stimulus to ensure that participants were relaxed. If $\mathrm{RMS}_{\mathrm{EMG}}$ was $>2$ standard deviations (SD) compared to mean baseline values, the evoked response following it was discarded. For that reason, SICI and ICF data from one participant were omitted from statistical analysis. $\mathrm{RMS}_{\mathrm{EMG}}$ data across all conditions and experiments are displayed in Table 1. Peak-to-peak amplitudes of the evoked responses were calculated. MEPs, LEPs and $H$-reflex peak-to-peak amplitudes were expressed as a percentage of peak-to-peak amplitudes of $M_{\max }\left(\mathrm{MEP} / M_{\max }, \mathrm{LEP} / M_{\max }\right.$, and $H / M_{\max }$, respectively). To quantify SICI and ICF, peak-to-peak amplitudes of unconditioned and conditioned MEPs were calculated, and the conditioned MEP amplitudes were expressed as a percentage of unconditioned MEP amplitudes.

\section{Statistical analyses}

All data are presented as mean \pm SD. Normality of data was assessed using Shapiro-Wilk test. If the data were not normally distributed, transformations were performed using common logarithm. A paired-sample $T$ test was used to assess differences in stimulus intensity at rMT (\% of stimulator output; SO) between SOL and TA. Sphericity was assessed using Mauchly's test of sphericity. In the case of violation, a Greenhouse-Geisser correction was employed. A repeated-measures ANOVA was used to assess differences in normalised evoked responses between resting position and passive shortening and lengthening (within-factor-a change in muscle length). Additional factor was added to ANOVA to assess differences between stimulations performed at different lengths (within-factor-muscle length at the point of stimulation). A two-way ANOVA was used to assess differences in fascicle length with passive ankle movement $(2 \times$ direction-shortening and lengthening; $5 \times$ joint angle). If significant $F$ values were found, analyses were continued using pairwise comparison with Bonferroni correction. In addition, Pearson's class correlation and a linear regression were performed to assess the association of intracortical facilitation or inhibition to a change in MEP/ $M_{\max }$ with a change in shortening or lengthening. Significance was set at an alpha level of 0.05 . All analyses were performed using SPSS (v20, SPSS Inc., Chicago, IL, USA). 


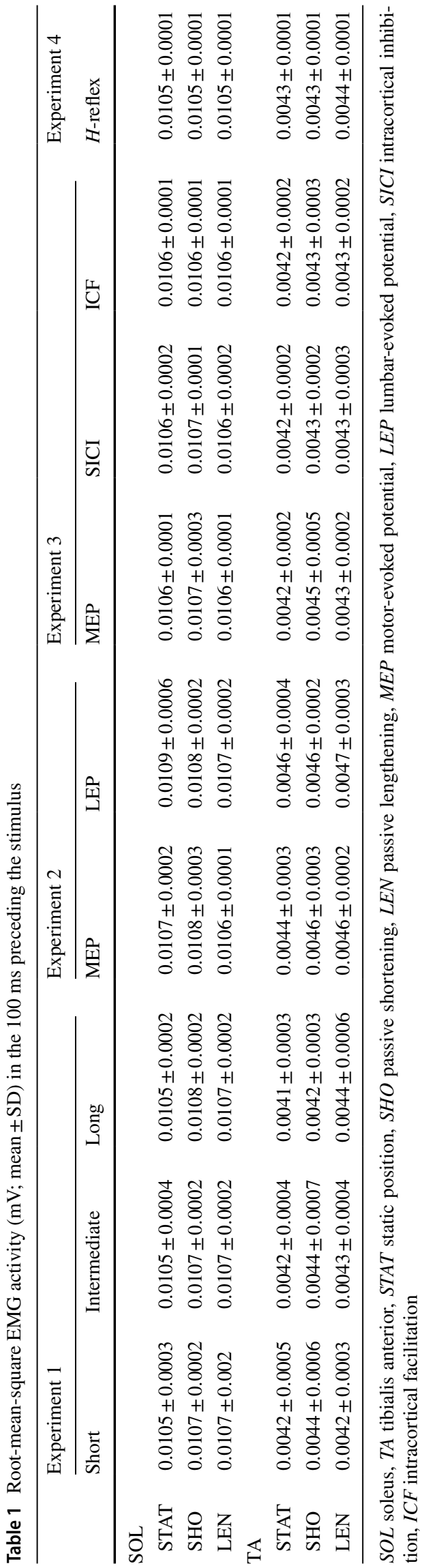

\section{Results}

\section{Fascicle length changes during passive ankle movement}

Fascicle length was modulated during passive ankle movement both in SOL $\left(F_{4,24}=109.9, p<0.001\right.$; Fig. 3a) and TA $\left(F_{4,24}=239.9, p<0.001\right.$; Fig. $\left.3 \mathrm{~b}\right)$, such that fascicle length changed linearly (Fig. 3 ) with changes in joint angle throughout the $20^{\circ}$ of range of motion $(p \leq 0.003$ and $p \leq 0.002$ for SOL and TA, respectively). Based on total change in fascicle length throughout the range of motion, the fascicles exhibited a similar mean change of $0.7 \mathrm{~mm} /{ }^{\circ}$ and $0.6 \mathrm{~mm} /{ }^{\circ}$ in SOL and TA, respectively $(p=0.388)$. In TA, fascicles were on average longer during passive lengthening $(40.9 \pm 4.1 \mathrm{~mm})$ compared to passive shortening $\left(39.4 \pm 4.5 \mathrm{~mm} ; F_{1,6}=10.3, p=0.018\right)$. However, no direction $\times$ angle interaction was found for both SOL $\left(F_{4,24}=1.5\right.$, $p=0.240)$ and TA $\left(F_{4,24}=1.2, p=0.357\right)$.

\section{Experiment 1: corticospinal responses at different muscle lengths during passive ankle movement}

The stimulus intensity at rMT was higher in SOL $(54 \pm 8 \%$ $\mathrm{SO})$ compared to TA ( $\left.48 \pm 7 \% \mathrm{SO} ; t_{11}=3.0, p=0.012\right)$. Examples of averaged EMG recordings in SOL (A) and TA (B) in response to single-pulse TMS are presented in Fig. 4. $\mathrm{MEP} / M_{\max }$ amplitude of SOL did not differ between the static position and during passive ankle movement ( $2 \pm 1$ vs. $2 \pm 1$ vs. $\left.2 \pm 1 \% M_{\max } ; F_{2,22}=2.3, p=0.121\right)$, irrespective of the joint angle at the point of stimulation ( $2 \pm 1$ vs. $2 \pm 1$ vs. $2 \pm 1 \% M_{\max }$ at short, intermediate and long muscle length, respectively; $F_{2,22}=0.2, p=0.787$; Fig. $4 c$ ). Conversely, a change in muscle length modulated MEP/ $M_{\max }$ amplitude in TA $\left(F_{1.3,14.6}=11.3, p=0.003\right)$ insofar as $\mathrm{MEP} / M_{\max }$ amplitude was greater during passive shortening $\left(17 \pm 9 \% M_{\max }\right)$ compared to passive lengthening $\left(9 \pm 7 \% M_{\max } ; p<0.001\right)$ and static position $\left(10 \pm 8 \% M_{\max } ; p=0.023\right.$; Fig. $\left.4 d\right)$, with no difference between passive lengthening and static position $(p=0.99)$. In addition, MEP/ $M_{\max }$ amplitude in TA was not affected by muscle length at the point of stimulation ( $12 \pm 9$ vs. $13 \pm 10$ vs. $11 \pm 7 \% M_{\max }$ at short, intermediate and long muscle length, respectively; $F_{2,22}=1.0, p=0.922$; Fig. 4d).

\section{Experiment 2: corticospinal and spinal motoneuronal responses during passive ankle movement}

The stimulus intensity at rMT was again higher in SOL $(49 \pm 9 \% \mathrm{SO})$ compared to TA $\left(46 \pm 10 \% \mathrm{SO} ; t_{10}=3.0\right.$, $p=0.014)$. Figure 5 shows the examples of averaged 
Fig. 3 Change in fascicle length in soleus and tibialis anterior with passive movement of the ankle. Fascicle length (mm) with passive changes in the ankle joint angle during passive shortening (left panel) and lengthening (right panel) of soleus (a) and tibialis anterior (b). Fascicle length was assessed at joint angles where stimuli were delivered in subsequent experiments and are displayed on the $x$-axes relative to anatomical zero (ankle at $90^{\circ}$ ). Fascicles changed linearly with changes in joint angle as noted on plots. Full lines represent the sample mean, whilst dashed lines denote individual responses $(n=7)$

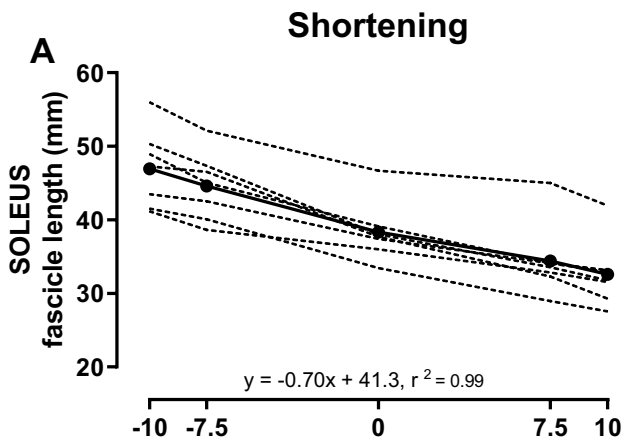

Lengthening

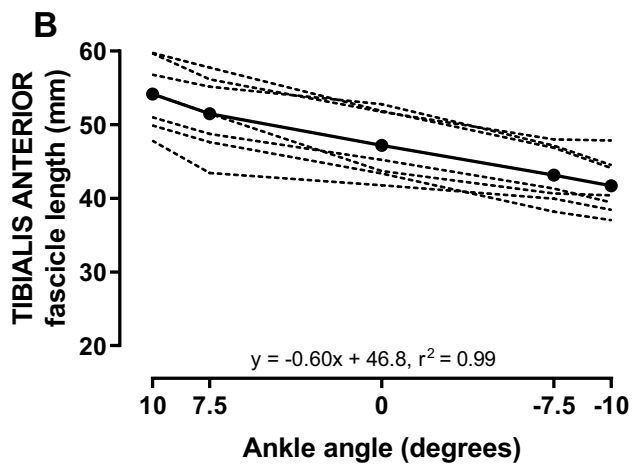

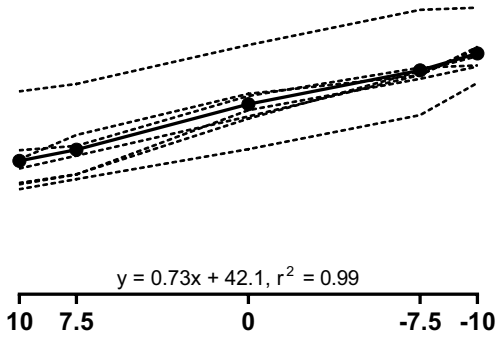
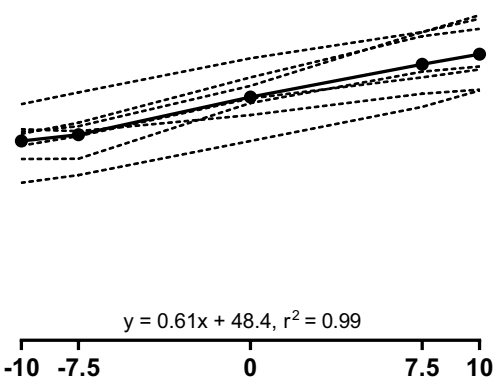

Ankle angle (degrees)
EMG recordings in SOL (A) and TA (B) in response to single-pulse TMS and electrical stimulation of descending axons at the lumbar spinal segments. These responses display similarities of MEPs in SOL across conditions (Fig. 5a). Both MEP/ $M_{\max }\left(2 \pm 1\right.$ vs. $2 \pm 2$ vs. $2 \pm 1 \% M_{\max }$; $\left.F_{2,20}=0.72, p=0.497\right)$ and $L E P / M_{\max }(7 \pm 2$ vs. $6 \pm 4$ vs. $4 \pm 3 \% M_{\max } ; F_{2,20}=2.95, p=0.075$ ) were not modulated in SOL during passive ankle movement (Fig. 5c). Similarly, LEP $/ M_{\max }$ did not change in TA with passive shortening and lengthening ( $9 \pm 3$ vs. $7 \pm 4$ vs. $11 \pm 6 \%$ $\left.M_{\max } ; F_{2,20}=3.63, p=0.071\right)$. However, MEP $/ M_{\text {max }}$ was modulated by a change in muscle length in TA $\left(F_{2,20}=14.67, p<0.001\right)$, being greater during passive shortening $\left(18 \pm 9 \% M_{\max }\right)$ compared to passive lengthening $\left(10 \pm 8 \% M_{\max } ; p=0.001\right)$ and static position $(9 \pm 5 \%$ $M_{\text {max }} ; p=0.003 ;$ Fig. 5d).

\section{Experiment 3: intracortical inhibition and facilitation during passive ankle movement}

The stimulus intensity rMT was higher in SOL $(51 \pm 12 \%$ $\mathrm{SO})$ compared to TA $\left(48 \pm 10 \% \mathrm{SO} ; t_{13}=4.5, p=0.001\right)$. $\mathrm{MEP} / M_{\max }$ in SOL was not modulated with a change in muscle length $\left(2 \pm 1\right.$ vs. $2 \pm 2$ vs. $2 \pm 1 \% M_{\max } ; F_{2,26}=1.65$, $p=0.211$; Fig. $6 \mathrm{a})$, but was in the TA $\left(F_{2,26}=15.96\right.$, $p<0.001$; Fig. 6b), such that it was greater during passive shortening $\left(21 \pm 14 \% M_{\max }\right)$ compared to passive lengthening $\left(12 \pm 11 \% M_{\max } ; p<0.001\right)$ and static position $(9 \pm 4 \%$ $\left.M_{\max } ; p=0.001\right)$. No modulation in SICI was observed in
SOL ( $71 \pm 22$ vs. $61 \pm 30$ vs. $60 \pm 23 \%$ unconditioned MEP; $\left.F_{2,26}=0.88, p=0.427\right)$ or in TA $(63 \pm 25$ vs. $55 \pm 23$ vs. $56 \pm 32 \%$ unconditioned MEP; $\left.F_{2,26}=0.63, p=0.540\right)$ during passive ankle movement, nor was ICF (SOL: $121 \pm 19$ vs. $134 \pm 38$ vs. $112 \pm 24 \%$ unconditioned MEP; $F_{2,26}=1.85$, $p=0.177$; TA: $129 \pm 37$ vs. $138 \pm 40$ vs. $145 \pm 61 \%$ unconditioned MEP; $\left.F_{2,26}=0.26, p=0.777\right)$. There was an inverse relationship between $\mathrm{MEP} / M_{\max }$ and ICF during passive shortening of TA ( $r=-0.625, p=0.017$, adjusted $\left.r^{2}=0.34\right)$, suggesting that greater corticospinal excitability observed during passive shortening was associated with a smaller degree of intracortical facilitation (Fig. 6c). No other associations were found between $\mathrm{MEP} / M_{\max }$ and SICI or ICF either in SOL or TA (Table 2).

\section{Experiment 4: $\mathrm{H}$-reflex during passive ankle movement}

Representative averaged traces of the $H$-reflex response from one individual are presented in Fig. 7a, b for SOL and TA, respectively. As clearly seen from these examples, the $H$-reflex responses were modulated during passive ankle movement in SOL $\left(F_{1.0 .4 .1}=8.4, p=0.043\right)$, being smaller during passive lengthening $\left(40 \pm 23 \% M_{\max }\right)$ compared to passive shortening $\left(56 \pm 17 \% M_{\max } ; p=0.048\right.$; Fig. $\left.7 \mathrm{c}\right)$. Conversely, $H / M_{\max }$ was not modulated during passive ankle movement in TA ( $4 \pm 3$ vs. $4 \pm 2$ vs. $5 \pm 3 \% M_{\max } ; F_{2,8}=1.6$, $p=0.258$; Fig. 7d). 


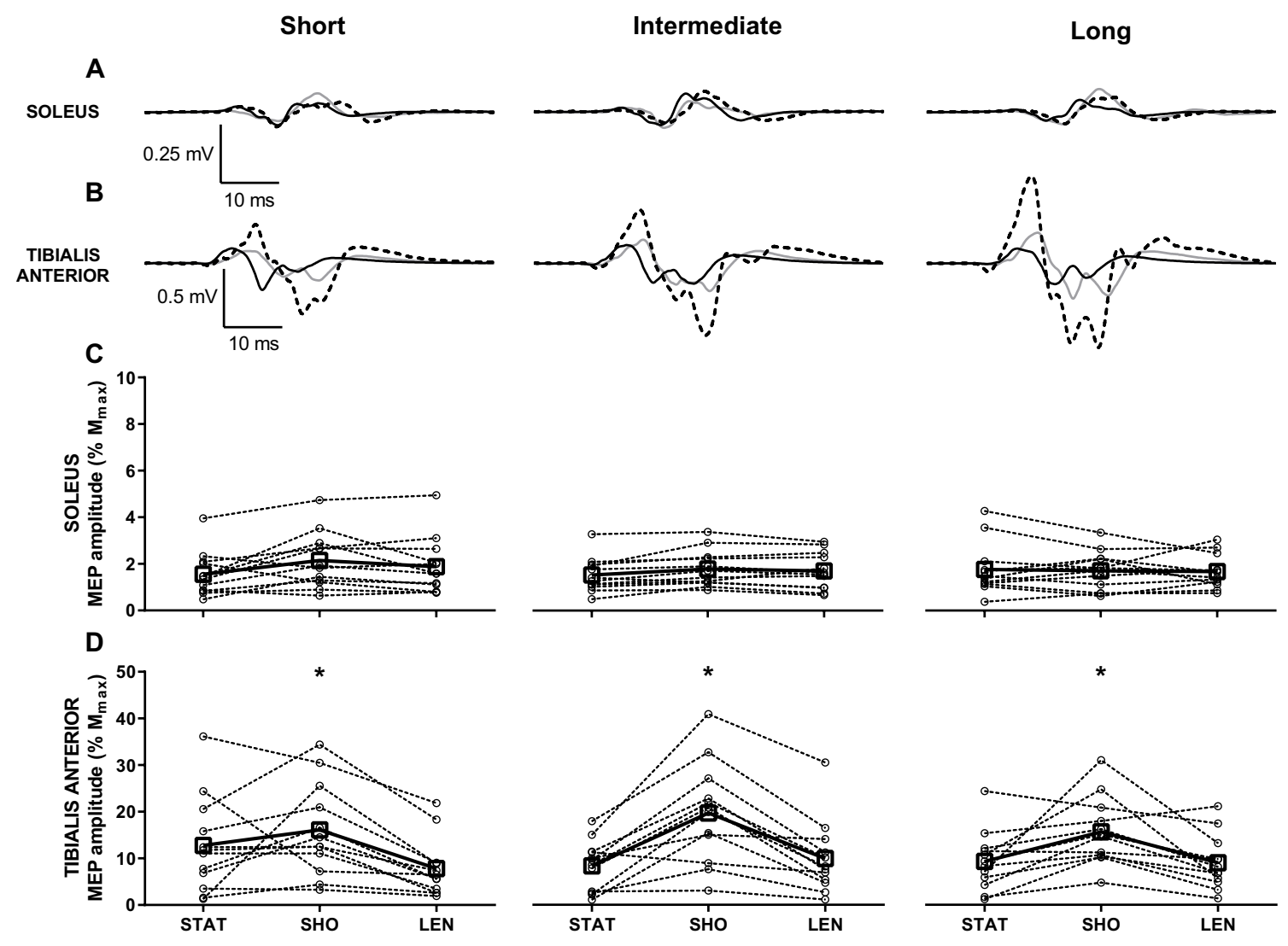

Fig. 4 Motor-evoked potentials during static position, passive shortening and lengthening in soleus, and tibialis anterior with stimuli delivered at different muscle length. a, b Averaged representative traces in response to single-pulse transcranial magnetic stimulation delivered at short, intermediate, and long muscle length during resting position (black line), passive shortening (grey line), and lengthening (dashed line) in soleus (a) and tibialis anterior (b). Each representative trace is an average of 15 waveforms. c, $\mathbf{d}$ Amplitude of motor-evoked potential expressed as a percentage of the amplitude of

\section{Discussion}

The main finding of this study was that corticospinal excitability is modulated differently between antagonist muscles during passive ankle movement. During passive movement, cortical excitability in TA was facilitated, but remained unchanged in SOL. Subcortical excitability at the lumbar spinal segmental level was not modulated in TA, suggesting a cortical and/or propriospinal contribution to the observed facilitation. These findings suggest a different intrinsic modulation of antagonist ankle muscles during passive movement.

\section{Modulation of corticospinal excitability during passive movement is not dependent on the muscle length at the point of stimulation}

The differing corticospinal response to TMS between the muscles cannot be attributed to muscle length change maximal compound action potential $\left(\mathrm{MEP} / M_{\max }\right)$ during static position (STAT), passive shortening (SHO), and passive lengthening (LEN) in soleus (c) and tibialis anterior (d) at short (left panel), intermediate (centre panel), and long (right panel) muscle length. Open squares and full lines represent the sample mean, whilst open circles and dashed lines denote individual responses $(n=12)$. $* p=0.023$ compared to static position, and $p<0.001$ compared to passive lengthening

differences, as both muscles exhibited a similar range of fascicle shortening and lengthening during passive movement ( 0.7 and $0.6 \mathrm{~mm} /{ }^{\circ}$ for SOL and TA, respectively). Contrary to our hypothesis, the responses were similar regardless of the muscle length at the point of stimulation. This contrasts also to the previous experiments of passive wrist movement (Lewis et al. 2001; Lewis and Byblow 2002) where corticospinal excitability was dependent on joint angle. However, direct comparison with the previous experiments is difficult, due to differences in muscles tested (upper limb vs. lower limb), ranges of motion, and different methodologies with regard to MEP amplitude normalisation. The latter might play a role in interpreting changes in response amplitude, since electrode position variations might lead to differences in the spatial relationship between the electrode and the motor units recorded (Farina et al. 2014), which is typically reflected in $M_{\max }$ amplitude (Gerilovsky et al. 1989). The range of motion, and the resultant muscle length changes, 


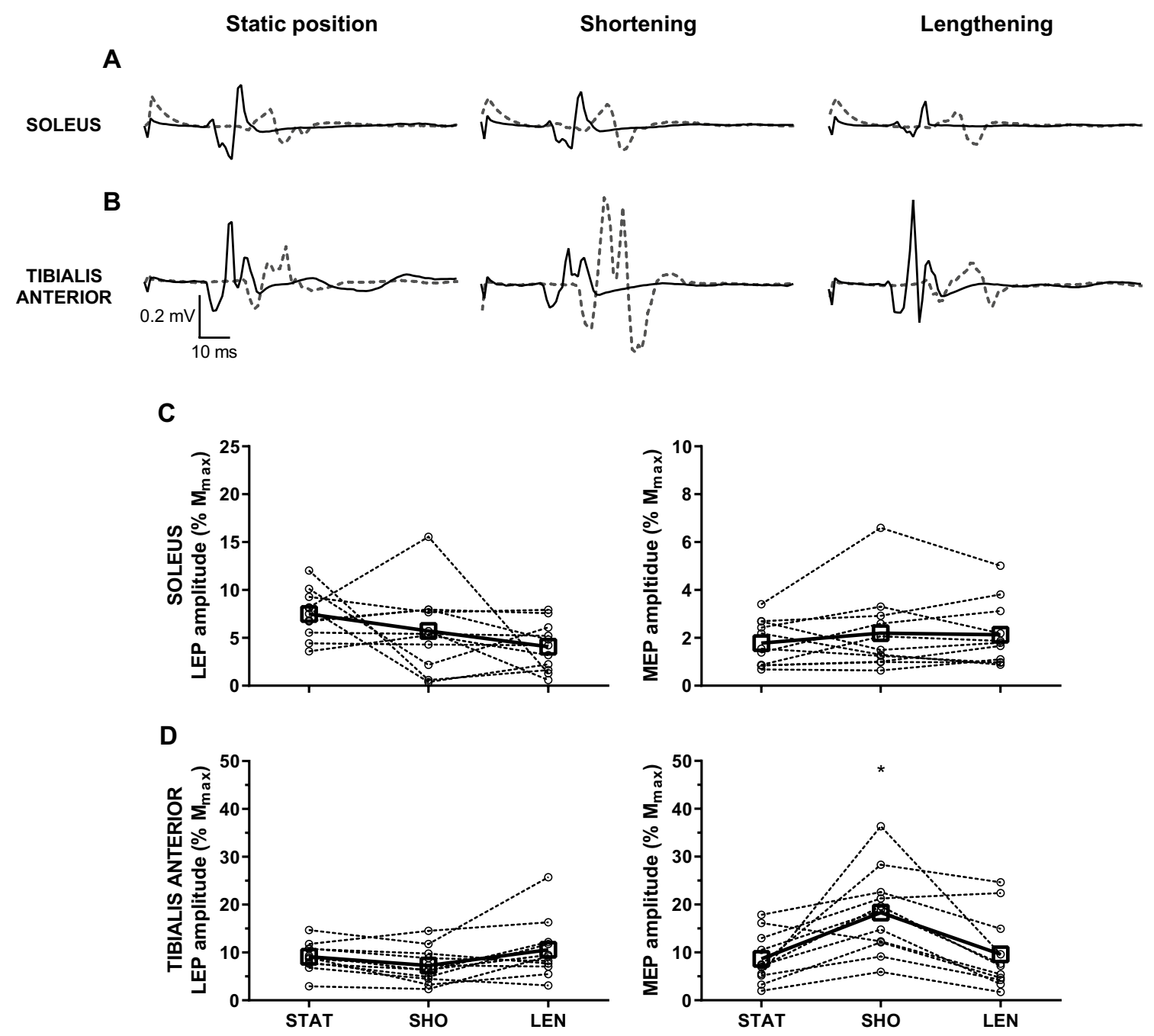

Fig. 5 Motor-evoked and lumbar-evoked potentials during static position, passive shortening, and lengthening in soleus and tibialis anterior. a, b Averaged representative traces in response to electrical stimulation of the lumbar spinous processes (black line) and single-pulse transcranial magnetic stimulation (dashed grey line) during static position (left panel), passive shortening (centre panel) and passive lengthening (right panel) in soleus (a) and tibialis anterior (b). Each representative trace is an average of ten waveforms. $\mathbf{c}, \mathbf{d}$ Amplitude

could be equally important in interpreting response amplitude. Indeed, a recent study in active knee extensors showed muscle length-dependent modulation of corticospinal excitability during lengthening contractions (Doguet et al. 2017). However, there was $\sim 11 \mathrm{~mm}$ fascicle length change when moving from an intermediate to long position (Doguet et al. 2018), compared to $\sim 5 \mathrm{~mm}$ seen in the present study. Thus, it seems plausible that there is a threshold of muscle length change after which increased afferent feedback is sufficient for detecting differences in corticospinal excitability. of lumbar-evoked potential (left panel) and motor-evoked potential (right panel) expressed as a percentage of the amplitude of maximal compound action potential (LEP/ $M_{\max }$ and $\mathrm{MEP} / M_{\max }$, respectively) during static position (STAT), passive shortening (SHO), and passive lengthening (LEN) in soleus (c) and tibialis anterior (d). Open squares and full lines represent the sample mean, whilst open circles and dashed lines denote individual responses $(n=11)$. ${ }^{*} p<0.005$ compared to static position and passive lengthening

\section{The responses to passive ankle movement are muscle specific}

The facilitation in corticospinal response to TMS observed in TA during shortening is in agreement with studies employing passive movement in the upper limb muscles (Lewis et al. 2001; Lewis and Byblow 2002; Coxon et al. 2005; Chye et al. 2010). As LEPs and $H$-reflexes remained unchanged in TA, this would suggest a cortical and/or propriospinal origin of facilitation. Conversely, corticospinal excitability in SOL 
A
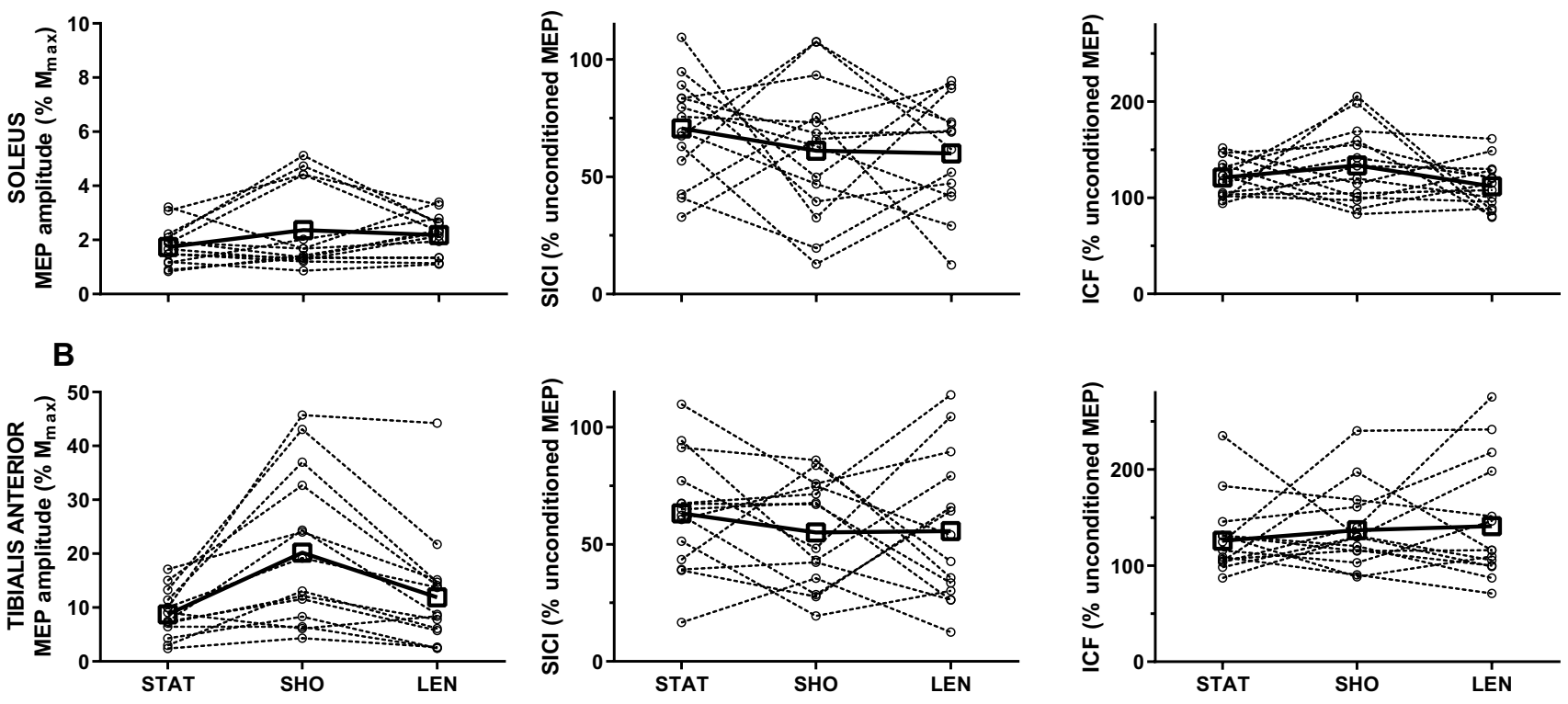

C

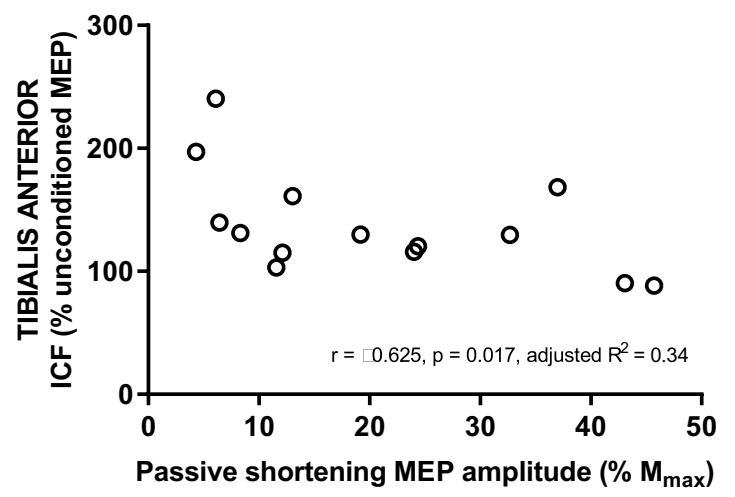

Fig. 6 Motor-evoked potentials evoked with single- and pairedpulse transcranial magnetic stimulation during static position, passive shortening, and lengthening in soleus and tibialis anterior. a, b Amplitude of motor-evoked potential expressed as a percentage of the amplitude of maximal compound action potential (MEP/ $M_{\max }$; left panel), short-interval intracortical inhibition (SICI; centre panel), and intracortical facilitation (ICF; right panel) expressed as a percentage of the unconditioned MEP amplitude during static position (STAT), passive shortening (SHO), and passive lengthening (LEN) in soleus

remained unchanged with passive movement. Due to lack of published data on corticospinal excitability during passive movement in SOL, no comparison can be made with the other studies. However, similar results have been obtained during active movement of SOL with comparable stimulus intensities (Duclay et al. 2011; Hahn et al. 2012; Valadão et al. 2018). Whilst LEPs remained unchanged in SOL, $H$-reflexes were reduced during passive lengthening. This latter finding corroborates the previous studies (Pinniger et al. 2001; Duclay et al. 2011), and has been attributed to presynaptic inhibition and post-activation depression of Ia afferents (a) and tibialis anterior (b). Open squares and full lines represent the sample mean, whilst open circles and dashed lines denote individual responses $(n=14)$. $* p<0.005$ compared to resting position and passive lengthening. $\mathbf{c}$ The amplitude of motor-evoked potential expressed as a percentage of the amplitude of maximal compound action potential $\left(\mathrm{MEP} / M_{\max }\right)$ plotted against ratio of conditioned and unconditioned motor-evoked potential amplitude (ICF) in response to paired-pulse transcranial magnetic stimulation with an inter-stimulus interval of $10 \mathrm{~ms}$ during passive shortening in TA $(n=14)$

(Hultborn et al. 1996). Given that LEPs are likely devoid of presynaptic influence (Nielsen and Petersen 1994), the lack of LEP modulation in SOL during passive movement further corroborates the notion that presynaptic inhibition mediates the reduction in $\mathrm{H}$-reflexes during passive lengthening.

\section{The activity of intracortical neurons during passive ankle movement}

The MEP $/ M_{\max }$ facilitation observed in TA during passive shortening was not accompanied by changes in responses 
Table 2 Associations between responses to single- and paired-pulse transcranial magnetic stimulation

\begin{tabular}{|c|c|c|c|c|c|c|c|c|c|c|c|c|}
\hline & \multicolumn{6}{|c|}{ SICI (/unconditioned MEP) } & \multicolumn{6}{|c|}{ ICF (/unconditioned MEP) } \\
\hline & \multicolumn{2}{|l|}{ STAT } & \multicolumn{2}{|l|}{$\mathrm{SHO}$} & \multicolumn{2}{|l|}{ LEN } & \multicolumn{2}{|l|}{ STAT } & \multicolumn{2}{|l|}{$\mathrm{SHO}$} & \multicolumn{2}{|l|}{ LEN } \\
\hline & $r$ & $p$ & $R$ & $p$ & $r$ & $p$ & $r$ & $p$ & $r$ & $p$ & $r$ & $p$ \\
\hline \multicolumn{13}{|l|}{$\mathrm{MEP} / M_{\max }$} \\
\hline \multicolumn{13}{|l|}{ SOL } \\
\hline STAT & -0.160 & 0.584 & - & - & - & - & 0.138 & 0.637 & - & - & - & - \\
\hline $\mathrm{SHO}$ & - & - & -0.042 & 0.887 & - & - & - & - & -0.143 & 0.626 & - & - \\
\hline LEN & - & - & - & - & -0.095 & 0.748 & - & - & - & - & -0.301 & 0.296 \\
\hline \multicolumn{13}{|l|}{ TA } \\
\hline STAT & -0.002 & 0.994 & - & - & - & - & -0.284 & 0.326 & - & - & - & - \\
\hline SHO & - & - & 0.077 & 0.794 & - & - & - & - & -0.625 & 0.017 & - & - \\
\hline LEN & - & - & - & - & -0.319 & 0.267 & - & - & - & - & -0.433 & 0.122 \\
\hline
\end{tabular}

$S O L$ soleus, TA tibialis anterior, STAT static position, $S H O$ passive shortening, $L E N$ passive lengthening, $M E P / M_{\max }$ motor-evoked potential normalised to maximal compound action potential, SICI intracortical inhibition, ICF intracortical facilitation, $r$ correlation coefficient, $p$ significance at alpha level 0.05

to paired-pulse TMS, and could be explained by greater response variability (see Fig. 6). This is a common occurrence and might be due to different electrophysiological properties of neuronal populations subserving the responses to SICI and ICF and inter-individual differences in synaptic efficacy of inhibitory or excitatory interneurons (Orth et al. 2003). It was also shown that the size of the MEP/ $M_{\max }$ during passive dorsiflexion negatively correlated with the ICF ratio, possibly due to the 'busy line' phenomenon, whereby glutamatergic circuitry activity is too high for conditioned MEPs to be facilitated (Ortu et al. 2008). Previous work has shown that SICI is modulated during passive wrist movements (Lewis et al. 2001), but is only evident at the transition from extension to flexion, and might be related to a sudden muscle length change and the corresponding initial burst in muscle spindle firing (Matthews 2011). When comparing responses elicited at similar joint angles, the lack of change in SICI corroborates the finding of the previous work (Lewis et al. 2001). Thus, the present data suggest that passive muscle length changes do not modulate cortical interneuronal activity.

\section{Cortical and propriospinal contribution to the observed corticospinal response}

Increased corticospinal excitability during passive shortening in TA in the absence of LEP modulation suggests a cortical origin, associated with sensory feedback influencing the excitability of descending tracts (Meinck and PiesiurStrehlow 1981; Roy and Gorassini 2008), or mediation via propriospinal inputs (Meinck and Piesiur-Strehlow 1981; Bestmann and Krakauer 2015).

In both primates (Hore et al. 1976; Herter et al. 2015) and humans (Goldring and Ratcheson 1972; Shaikhouni et al.
2013), cortical neurons have been shown to be facilitated during passive shortening, whilst inhibited during passive lengthening, which agrees with our findings. Cutaneous and joint receptors are unlikely mediators of this behaviour due to their activation being largely restricted to the limits of movement (Burke et al. 1988), rather than throughout the movement. Thus, the primary candidates for the sensory mediated change in cortical neuronal activity are muscle spindle afferents. This mediation might involve inhibitory inputs, either directly to motor cortical areas or through the somatosensory cortex. Indeed, in primates, hindlimb muscle stretch has been shown to result in inhibition of area 4 cortical neurons due to direct input from group II afferents (Hore et al. 1976). Furthermore, changes in TA muscle fascicle length have been shown to be tightly linked to Ia afferent sensitivity in humans (Day et al. 2017). Thus, increased corticospinal responses during passive shortening of TA might stem from decreased Ia afferent input via area 3a of the cerebral cortex (Hore et al. 1976), resulting in disinhibition of corticospinal neurons, and, thus, increasing corticospinal excitability (Brasil-Neto et al. 1992; Ziemann et al. 1998).

It is unclear why the augmented corticospinal response to TMS during passive shortening is specific to TA. It might stem from divergent, non-uniform distribution of direct corticomotoneuronal projections, as evidenced by short latency facilitation of firing probability of TA motor units in response to TMS, and the absence of this behaviour in SOL (Brouwer and Ashby 1992; Brouwer and Qiao 1995). This could have contributed to the facilitation of TA during passive shortening when corticospinal neurons may be disinhibited relative to passive lengthening (Brasil-Neto et al. 1992; Ziemann et al. 1998). There is some basis for this notion as greater facilitation during passive shortening has been observed in the wrist muscles with greater strength 

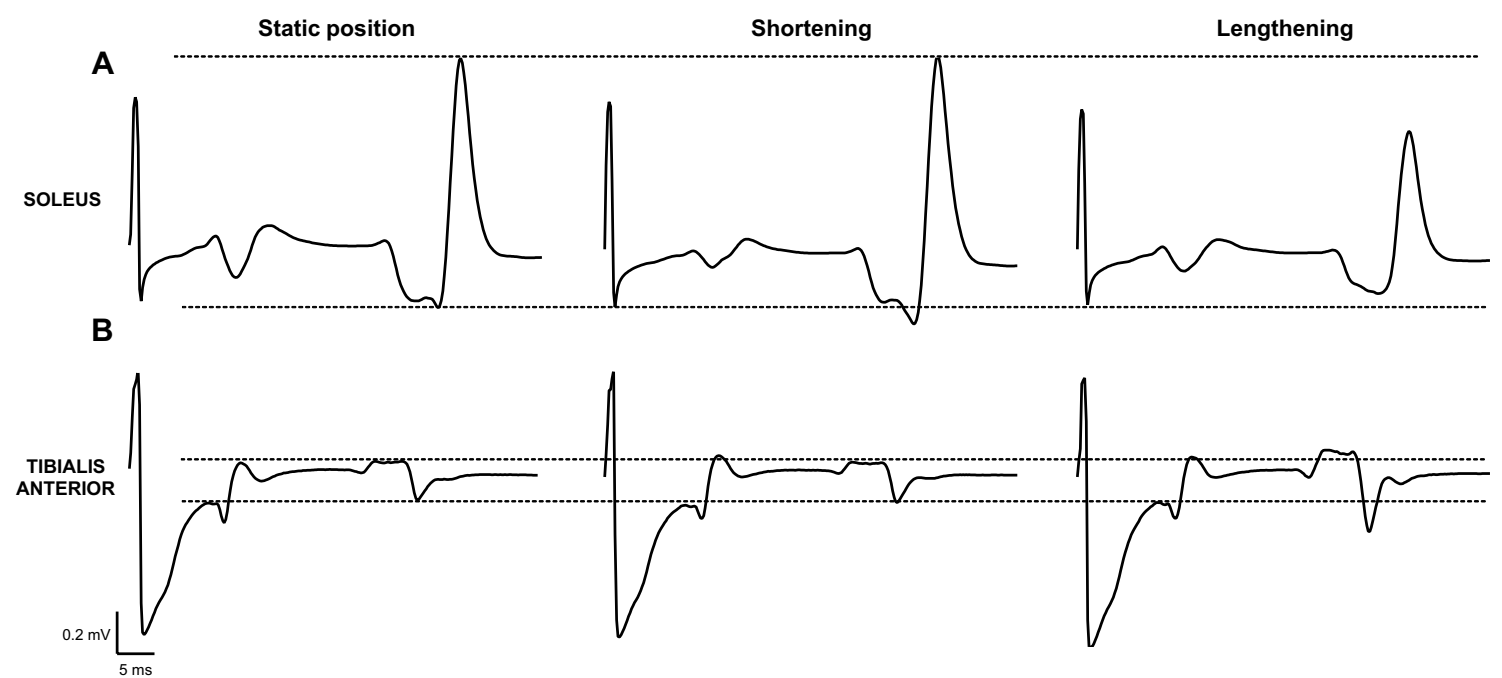

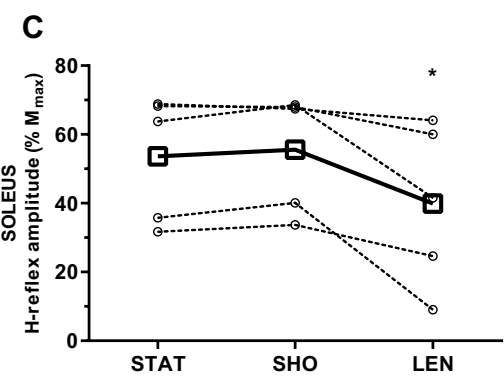

Fig. $7 H$-reflexes during static position, passive shortening, and lengthening in soleus and tibialis anterior. a, b Averaged representative traces in response to submaximal percutaneous nerve stimulation during static position (left panel), passive shortening (centre panel), and passive lengthening (right panel) in soleus (a) and tibialis anterior (b). Traces are shown from the point of stimulus and each representative trace is an average of ten responses. Dashed lines represent

of corticomotoneuronal projections (Chye et al. 2010). The pyramidal tract also has a preferential input into the spinal network controlling ankle flexors, such as TA (Brooks and Stoney 1971), which could explain the lower stimulus intensity at rMT in the present study whilst also supporting the previous work (Lauber et al. 2018). In addition, the responses in TA could be related to differing reciprocal inhibition compared to SOL (Yavuz et al. 2018). Less reciprocal inhibition as SOL lengthens would suppress the excitatory postsynaptic potential stemming from the antagonist, allowing for reduced inhibition in corticospinal neurons in TA. Furthermore, as per $H$-reflex behaviour in the present study, TA appears to be influenced by presynaptic inhibitory mechanisms to a lesser extent than SOL. Thus, the facilitation observed during passive shortening of TA could be due to coupling of the lack of presynaptic influences and sensoryrelated facilitation of corticospinal excitability in response to movement (Schubert et al. 1997). Nonetheless, it should be noted that despite the plausibility of the above-mentioned
D

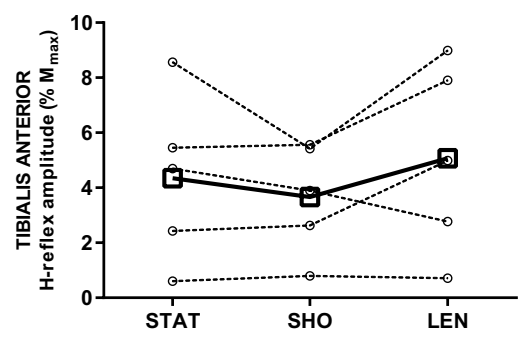

the amplitude of $H$-reflex during static position. c, d Amplitude of $H$-reflex expressed as a percentage of the amplitude of maximal compound action potential during static position (STAT), passive shortening (SHO), and passive lengthening (LEN) in soleus (c) and tibialis anterior (d). Open squares and full lines represent the sample mean, whilst open circles and dashed lines denote individual responses $(n=5) . * p<0.05$ compared to passive shortening

notions, this study cannot directly ascertain the mechanism of the observed behaviour.

An increase in presynaptic inhibitory input to alpha motoneurons was observed during passive lengthening of SOL, with no accompanying change in MEPs and LEPs. This suggests a form of compensatory action of descending pathways during passive SOL lengthening to accommodate for reduced motoneuronal excitability. Given a lack of change in ICF and SICI, this compensation is unlikely to be intracortical in origin, pointing to the possibility of propriospinal mediation. This could occur through facilitation of excitatory premotoneurons activated by group II afferents (Marque et al. 2005), which are likely to exhibit increased firing rate during muscle lengthening (Matthews 2011). The specificity of this compensation to SOL is less clear, but it might again be related to asymmetrical distribution of reciprocal inhibitory input between TA and SOL (Yavuz et al. 2018). 


\section{Potential functional applications of the observed behaviour}

The specificity of augmented corticospinal response in TA relative to SOL during passive shortening could reflect functional differences between these muscles. For example, during quiet standing, TA has been shown to exhibit passive fascicle length changes proportional to the sway-related changes in the ankle joint (Di Giulio et al. 2009; Day et al. 2013). The present data might, thus, suggest an important role of increasing corticospinal drive in this muscle during passive shortening when proprioceptive feedback originating from muscle spindles is reduced, to modulate the control signals of the antagonist via reciprocal inhibition (Di Giulio et al. 2009; Honeycutt et al. 2012).

\section{Methodological considerations}

The lack of modulation of corticospinal excitability during passive movement of the ankle in SOL could be due to the slow movement velocity used in the present study. Indeed, the previous work using higher movement velocities has shown greater modulation in response size (Lewis et al. 2001; Lewis and Byblow 2002), likely due to higher afferent feedback. The slower velocity was employed to ensure greater ability of relaxation and to avoid reflexive muscle activity related to passive movement, which could have confounded results (Pinniger et al. 2001). Furthermore, the relatively smaller ankle range of motion in the present experiment reflects the restriction and variability in joint mobility, particularly at dorsiflexion. In the upper limb, the previous research has shown potentiated effects on corticospinal excitability during passive movement with greater ranges of motion (Coxon et al. 2005). Thus, future studies should explore the velocity- and muscle-length dependence of the responses.

Other limitations of the present study are the lack of repeated-measures design and a small sample size in Experiment 4 . With regard to the former, the significant facilitation of the response to TMS during passive shortening of TA was replicated across three experiments (Experiment 1-3), suggesting a universal behaviour across different sample populations. As already noted, there was difficulty in obtaining $H$-reflexes in resting TA, corroborating the previous reports (Roy and Gorassini 2008; Burke 2016). Despite screening 24 individuals, only five participants exhibited consistent $H$-reflexes in TA to allow for comparison with SOL. This small sample size does warrant caution in interpreting the findings of Experiment 4. However, the SOL data corroborates the findings of previous work (Pinniger et al. 2001) and suggests that presynaptic inhibition during passive lengthening is greater compared to TA.

\section{Conclusions}

As hypothesised, the segmental methodological approach revealed that changing muscle length modulates both corticospinal and spinal elements of the nervous system during passive movement, but is muscle specific. Contrary to our hypothesis, the corticospinal modulation occurred regardless of the muscle length at the point of assessment. Corticospinal excitability was facilitated in TA during passive shortening, whilst unmodulated in SOL. This suggests that neural modulation with movement should be interpreted in the context of the muscle investigated. During muscle shortening, a reduced inhibitory afferent input might explain the flexor-biased facilitation in corticospinal drive.

Acknowledgements The authors thank Mr. Tom Pearson of Cambridge Electronics Design Ltd. for designing scripts that facilitated data analysis.

Open Access This article is distributed under the terms of the Creative Commons Attribution 4.0 International License (http://creativeco mmons.org/licenses/by/4.0/), which permits unrestricted use, distribution, and reproduction in any medium, provided you give appropriate credit to the original author(s) and the source, provide a link to the Creative Commons license, and indicate if changes were made.

\section{References}

Abbruzzese G, Morena M, Spadavecchia L, Schieppati M (1994) Response of arm flexor muscles to magnetic and electrical brain stimulation during shortening and lengthening tasks in man. $\mathbf{J}$ Physiol 481:499-507

Ando R, Taniguchi K, Saito A et al (2014) Validity of fascicle length estimation in the vastus lateralis and vastus intermedius using ultrasonography. J Electromyogr Kinesiol 24:214-220. https:// doi.org/10.1016/j.jelekin.2014.01.003

Ansdell P, Brownstein CG, Škarabot J et al (2019) Menstrual cycle associated modulations in neuromuscular function and fatigability of the knee extensors in eumenorrheic females. J Appl Physiol 126:1701-1712. https://doi.org/10.1152/japplphysi ol.01041.2018

Banks RW (2006) An allometric analysis of the number of muscle spindles in mammalian skeletal muscles. J Anat 208:753-768. https://doi.org/10.1111/j.1469-7580.2006.00558.x

Barrué-Belou S, Marque P, Duclay J (2018) Recurrent inhibition is higher in eccentric compared to isometric and concentric maximal voluntary contractions. Acta Physiol (Oxf) 223:e13064. https:// doi.org/10.1111/apha.13064

Bestmann S, Krakauer JW (2015) The uses and interpretations of the motor-evoked potential for understanding behaviour. Exp Brain Res 233:679-689. https://doi.org/10.1007/s00221-014-4183-7

Bland DC, Prosser LA, Bellini LA et al (2011) Tibialis anterior architecture, strength, and gait in individuals with cerebral palsy. Muscle Nerve 44:509-517. https://doi.org/10.1002/mus.22098

Brasil-Neto JP, Cohen LG, Pascual-Leone A et al (1992) Rapid reversible modulation of human motor outputs after transient deafferentation of the forearm: a study with transcranial magnetic stimulation. Neurology 42:1302-1306 
Brooks VB, Stoney SD (1971) Motor mechanisms: the role of the pyramidal system in motor control. Annu Rev Physiol 33:337388. https://doi.org/10.1146/annurev.ph.33.030171.002005

Brouwer B, Ashby P (1990) Corticospinal projections to upper and lower limb spinal motoneurons in man. Electroencephalogr Clin Neurophysiol 76:509-519

Brouwer B, Ashby P (1992) Corticospinal projections to lower limb motoneurons in man. Exp Brain Res 89:649-654

Brouwer B, Qiao J (1995) Characteristics and variability of lower limb motoneuron responses to transcranial magnetic stimulation. Electroencephalogr Clin Neurophysiol 97:49-54

Brownstein C, Ansdell P, Škarabot J et al (2018) An optimal protocol for measurement of corticospinal excitability, short intracortical inhibition and intracortical facilitation in the rectus femoris. J Neurol Sci 394:45-56. https://doi.org/10.1016/j.jns.2018.09.001

Burke RE (1967) Motor unit types of cat triceps surae muscle. J Physiol 193:141-160

Burke D (2016) Clinical uses of $\mathrm{H}$ reflexes of upper and lower limb muscles. Clin Neurophysiol Pract 1:9-17. https://doi. org/10.1016/J.CNP.2016.02.003

Burke D, Gandevia SC, Macefield G (1988) Responses to passive movement of receptors in joint, skin and muscle of the human hand. J Physiol 402:347-361

Byrne CA, O'Keeffe DT, Donnelly AE, Lyons GM (2007) Effect of walking speed changes on tibialis anterior EMG during healthy gait for FES envelope design in drop foot correction. J Electromyogr Kinesiol 17:605-616

Capaday C, Lavoie BA, Barbeau H et al (1999) Studies on the corticospinal control of human walking. I. Responses to focal transcranial magnetic stimulation of the motor cortex. J Neurophysiol 81:129-139. https://doi.org/10.1152/jn.1999.81.1.129

Chen R, Tam A, Bütefisch C et al (1998) Intracortical inhibition and facilitation in different representations of the human motor cortex. J Neurophysiol 80:2870-2881

Chye L, Nosaka K, Murray L et al (2010) Corticomotor excitability of wrist flexor and extensor muscles during active and passive movement. Hum Mov Sci 29:494-501. https://doi.org/10.1016/j. humov.2010.03.003

Coren S (1993) The lateral preference inventory for measurement of handedness, footedness, eyedness, and earedness: norms for young adults. Bull Psychon Soc 31:1-3. https://doi.org/10.3758/BF033 34122

Coxon JP, Stinear JW, Byblow WD (2005) Amplitude of muscle stretch modulates corticomotor gain during passive movement. Brain Res 1031:109-117. https://doi.org/10.1016/j.brainres.2004.10.062

Day JT, Lichtwark GA, Cresswell AG (2013) Tibialis anterior muscle fascicle dynamics adequately represent postural sway during standing balance. J Appl Physiol 115:1742-1750. https://doi. org/10.1152/japplphysiol.00517.2013

Day J, Bent LR, Birznieks I et al (2017) Muscle spindles in human tibialis anterior encode muscle fascicle length changes. J Neurophysiol 117:1489-1498. https://doi.org/10.1152/jn.00374.2016

De Luca CJ, Kline JC (2012) Influence of proprioceptive feedback on the firing rate and recruitment of motoneurons. J Neural Eng 9:016007. https://doi.org/10.1088/1741-2560/9/1/016007

Devanne H, Lavoie BA, Capaday C (1997) Input-output properties and gain changes in the human corticospinal pathway. Exp Brain Res 114:329-338. https://doi.org/10.1007/PL00005641

Di Giulio I, Maganaris CN, Baltzopoulos V, Loram ID (2009) The proprioceptive and agonist roles of gastrocnemius, soleus and tibialis anterior muscles in maintaining human upright posture. J Physiol 587:2399-2416. https://doi.org/10.1113/jphysiol.2009.168690

Doguet V, Nosaka K, Guével A et al (2017) Muscle length effect on corticospinal excitability during maximal concentric, isometric and eccentric contractions of the knee extensors. Exp Physiol 102:1513-1523. https://doi.org/10.1113/EP086480
Doguet V, Nosaka K, Guével A, Jubeau M (2018) Reply to the Letter to the Editor: Comments on Doguet et al. (2017) 'Muscle length effect on corticospinal excitability during maximal concentric, isometric and eccentric contractions of the knee extensors'. Exp Physiol 103:1437-1438. https://doi.org/10.1113/ep087248

Duclay J, Martin A (2005) Evoked H-reflex and V-wave responses during maximal isometric, concentric, and eccentric muscle contraction. J Neurophysiol 94:3555-3562. https://doi.org/10.1152/ jn.00348.2005

Duclay J, Pasquet B, Martin A, Duchateau J (2011) Specific modulation of corticospinal and spinal excitabilities during maximal voluntary isometric, shortening and lengthening contractions in synergist muscles. J Physiol 589:2901-2916. https://doi.org/10.1113/jphys iol.2011.207472

Duclay J, Pasquet B, Martin A, Duchateau J (2014) Specific modulation of spinal and cortical excitabilities during lengthening and shortening submaximal and maximal contractions in plantar flexor muscles. J Appl Physiol 117:1440-1450. https://doi.org/10.1152/ japplphysiol.00489.2014

Dum RP, Kennedy TT (1980) Synaptic organization of defined motorunit types in cat tibialis anterior. J Neurophysiol 43:1631-1644. https://doi.org/10.1152/jn.1980.43.6.1631

Elliott K, Cable N, Reilly T, Diver M (2003) Effect of menstrual cycle phase on the concentration of bioavailable 17- $\beta$ oestradiol and testosterone and muscle strength. Clin Sci 105:663-669. https:// doi.org/10.1042/CS20020360

Farina D, Merletti R, Enoka RM (2014) The extraction of neural strategies from the surface EMG: an update. J Appl Physiol 117:12151230. https://doi.org/10.1152/japplphysiol.00162.2014

Faul F, Erdfelder E, Lang A-G, Buchner A (2007) G*Power 3: a flexible statistical power analysis program for the social, behavioral, and biomedical sciences. Behav Res Methods 39:175-191

Gerilovsky L, Tsvetinov P, Trenkova G (1989) Peripheral effects on the amplitude of monopolar and bipolar H-reflex potentials from the soleus muscle. Exp Brain Res 76:173-181

Goldring S, Ratcheson R (1972) Human motor cortex: sensory input data from single neuron recordings. Science 175:1493-1495

Gruber M, Linnamo V, Strojnik V et al (2009) Excitability at the motoneuron pool and motor cortex is specifically modulated in lengthening compared to isometric contractions. J Neurophysiol 101:2030-2040

Guilhem G, Cornu C, Guével A (2011) Muscle architecture and EMG activity changes during isotonic and isokinetic eccentric exercises. Eur J Appl Physiol 111:2723-2733. https://doi.org/10.1007/s0042 1-011-1894-3

Hahn D, Hoffman BW, Carroll TJ, Cresswell AG (2012) Cortical and spinal excitability during and after lengthening contractions of the human plantar flexor muscles performed with maximal voluntary effort. PLoS One 7:e49907. https://doi.org/10.1371/journ al.pone.0049907

Han TR, Kim JH, Lim JY (2001) Optimization of facilitation related to threshold in transcranial magnetic stimulation. Clin Neurophysiol 112:593-599

Hermens HJ, Freriks B, Disselhorst-Klug C, Rau G (2000) Development of recommendations for SEMG sensors and sensor placement procedures. J Electromyogr Kinesiol 10:361-374

Herter TM, Takei T, Munoz DP, Scott SH (2015) Neurons in red nucleus and primary motor cortex exhibit similar responses to mechanical perturbations applied to the upper-limb during posture. Front Integr Neurosci 9:29. https://doi.org/10.3389/fnint .2015 .00029

Honeycutt CF, Nardelli P, Cope TC, Nichols TR (2012) Muscle spindle responses to horizontal support surface perturbation in the anesthetized cat: insights into the role of autogenic feedback in whole body postural control. J Neurophysiol 108:1253-1261. https://doi. org/10.1152/jn.00929.2011 
Hore J, Preston JB, Cheney PD (1976) Responses of cortical neurons (areas 3a and 4) to ramp stretch of hindlimb muscles in the baboon. J Neurophysiol 39:484-500. https://doi.org/10.1152/ jn.1976.39.3.484

Hultborn H, Illert M, Nielsen J et al (1996) On the mechanism of the post-activation depression of the H-reflex in human subjects. Exp Brain Res 108:450-462

Keel JC, Smith MJ, Wassermann EM (2001) A safety screening questionnaire for transcranial magnetic stimulation. Clin Neurophysiol 112:720

Kuhn A, Keller T, Lawrence M, Morari M (2010) The influence of electrode size on selectivity and comfort in transcutaneous electrical stimulation of the forearm. IEEE Trans Neural Syst Rehabil Eng 18:255-262. https://doi.org/10.1109/TNSRE.2009.2039807

Lauber B, Gollhofer A, Taube W (2018) Differences in motor cortical control of the Soleus and Tibialis. J Exp Biol. https://doi. org/10.1242/jeb. 174680

Lewis GN, Byblow WD (2002) Modulations in corticomotor excitability during passive upper-limb movement: is there a cortical influence? Brain Res 943:263-275

Lewis GN, Byblow WD, Carson RG (2001) Phasic modulation of corticomotor excitability during passive movement of the upper limb: effects of movement frequency and muscle specificity. Brain Res 900:282-294

Marque P, Nicolas G, Simonetta-Moreau M et al (2005) Group II excitations from plantar foot muscles to human leg and thigh motoneurons. Exp Brain Res 161:486-501. https://doi.org/10.1007/ s00221-004-2096-6

Matthews PBC (2011) Muscle spindles: their messages and their fusimotor supply. Compr Physiol (Supplement 2: Handbook of Physiology, The Nervous System, Motor Control):189-228. https ://doi.org/10.1002/cphy.cp010206 (first published in print 1981)

Matthews PB, Stein RB (1969) The sensitivity of muscle spindle afferents to small sinusoidal changes of length. J Physiol 200:723-743

Meinck HM, Piesiur-Strehlow B (1981) Reflexes evoked in leg muscles from arm afferents: a propriospinal pathway in man? Exp Brain Res 43:78-86

Morgan DL, Whitehead NP, Wise AK et al (2000) Tension changes in the cat soleus muscle following slow stretch or shortening of the contracting muscle. J Physiol 522(Pt 3):503-513

Morita H, Olivier E, Baumgarten J et al (2000) Differential changes in corticospinal and Ia input to tibialis anterior and soleus motor neurones during voluntary contraction in man. Acta Physiol Scand 170:65-76. https://doi.org/10.1046/j.1365-201x.2000.00762.x

Nielsen J, Petersen N (1994) Is presynaptic inhibition distributed to corticospinal fibres in man? J Physiol 477:47-58. https://doi. org/10.1113/jphysiol.1994.sp020170

Nielsen JB, Pyndt HS, Petersen NT (2003) Investigating human motor control by transcranial magnetic stimulation. Exp Brain Res 152:1-16. https://doi.org/10.1007/s00221-003-1537-y

Orth M, Snijders AH, Rothwell JC (2003) The variability of intracortical inhibition and facilitation. Clin Neurophysiol 114:2362-2369

Ortu E, Deriu F, Suppa A et al (2008) Effects of volitional contraction on intracortical inhibition and facilitation in the human motor cortex. J Physiol 586:5147-5159. https://doi.org/10.1113/jphys iol.2008.158956
Pierrot-Deseilligny E, Burke D (2005) The circuitry of the human spinal cord: its role in motor control and movement disorders. Cambridge University Press, Cambridge

Pinniger GJ, Nordlund M, Steele JR, Cresswell AG (2001) H-reflex modulation during passive lengthening and shortening of the human triceps surae. J Physiol 534:913-923

Proske U, Morgan D, Gregory J (1993) Thixotropy in skeletal muscle and in muscle spindles: a review. Prog Neurobiol 41:705-721

Reeves ND, Narici MV (2003) Behavior of human muscle fascicles during shortening and lengthening contractions in vivo. J Appl Physiol 95:1090-1096. https://doi.org/10.1152/japplphysi ol.01046.2002

Rossini PM, Barker AT, Berardelli A et al (1994) Non-invasive electrical and magnetic stimulation of the brain, spinal cord and roots: basic principles and procedures for routine clinical application. Report of an IFCN committee. Electroencephalogr Clin Neurophysiol 91:79-92

Roy FD, Gorassini MA (2008) Peripheral sensory activation of cortical circuits in the leg motor cortex of man. J Physiol 586:4091-4105. https://doi.org/10.1113/jphysiol.2008.153726

Schubert M, Curt A, Jensen L, Dietz V (1997) Corticospinal input in human gait: modulation of magnetically evoked motor responses. Exp Brain Res 115:234-246

Shaikhouni A, Donoghue JP, Hochberg LR (2013) Somatosensory responses in a human motor cortex. J Neurophysiol 109:21922204. https://doi.org/10.1152/jn.00368.2012

Škarabot J, Ansdell P, Brownstein CG et al (2018) Electrical stimulation of human corticospinal axons at the level of the lumbar spinal segments. Eur J Neurosci 49:1254-1267. https://doi.org/10.1111/ ejn. 14321

Smith M-C, Stinear JW, Alan Barber P, Stinear CM (2017) Effects of non-target leg activation, TMS coil orientation, and limb dominance on lower limb motor cortex excitability. Brain Res 1655:1016. https://doi.org/10.1016/j.brainres.2016.11.004

Ugawa Y, Genba-Shimizu K, Kanazawa I (1995) Electrical stimulation of the human descending motor tracts at several levels. Can J Neurol Sci 22:36-42

Valadão P, Kurokawa S, Finni T, Avela J (2018) Effects of muscle action type on corticospinal excitability and triceps surae muscle-tendon mechanics. J Neurophysiol 119:563-572. https://doi. org/10.1152/jn.00079.2017

Yavuz UŞ, Negro F, Diedrichs R, Farina D (2018) Reciprocal inhibition between motor neurons of the tibialis anterior and triceps surae in humans. J Neurophysiol 119:1699-1706. https://doi.org/10.1152/ jn.00424.2017

Ziemann U, Corwell B, Cohen LG (1998) Modulation of plasticity in human motor cortex after forearm ischemic nerve block. J Neurosci 18:1115-1123

Publisher's Note Springer Nature remains neutral with regard to jurisdictional claims in published maps and institutional affiliations. 